\title{
Institutional or individual experience matters in minimally invasive valve surgery
}

\author{
Robert B. Hawkins, MD, and Gorav Ailawadi, MD
}

\footnotetext{
From the Division of Thoracic and Cardiovascular Surgery, Department of Surgery, University of Virginia, Charlottesville, Va.

Disclosures: G.A. reports modest consulting fees from Abbott Vascular, Mitralign, and Edwards, and modest speaker fees from St Jude Medical. R.B.H. has nothing to disclose with regard to commercial support.

Received for publication July 7, 2016; accepted for publication July 12, 2016; available ahead of print Aug 16 , 2016.

Address for reprints: Gorav Ailawadi, MD, Department of Surgery, University of Virginia, PO Box 800679 , Charlottesville, VA 22908 (E-mail: gorav@ virginia.edu).

J Thorac Cardiovasc Surg 2016;152:1477-8

$0022-5223 / \$ 36.00$

Copyright (C) 2016 Published by Elsevier Inc. on behalf of The American Association for Thoracic Surgery http://dx.doi.org/10.1016/j.jtcvs.2016.07.027
}

Murzi and colleagues ${ }^{1}$ are to be congratulated for systematically analyzing the introduction of new technology at their institution. We applaud the rigorous methods, using the cumulative sum control chart (CUSUM) technique, used to ensure quality and patient safety that are often overlooked with the introduction of continuously evolving technology. They conclude that a minithoracotomy approach with a sutureless aortic valve implant can be used safely "without learning curve effects."

Minimally invasive cardiac surgery is driven by both patient preference and improved outcomes. Transitioning to minimally invasive approaches can be challenging, and analyses at high-volume centers have elucidated a clear, although variable, learning curve. ${ }^{2}$ Murzi and colleagues ${ }^{3}$ previously described their experience with aortic valve replacement (AVR) via a right anterior minithoracotomy (RAMT), which incurred longer crossclamp and bypass times, although no clear learning curve in the single surgeon analysis.

Technologic advancement can be disruptive or incremental, with disruptive advances gaining all the headlines. However, this study helps to shed light on the importance of incremental changes that decrease procedural complexity. The addition of sutureless valve technology to minimally invasive AVR is a logical step. Compared with traditional prosthesis implantation via RAMT, the transition to a sutureless valve resulted in shorter crossclamp and bypass times. ${ }^{4}$

In addition to the improved outcomes, the current study demonstrated no learning curve on an individual surgeon level by CUSUM analysis. However, there was variability in outcomes across the 6 participating surgeons, 3 of whom demonstrated some evidence of a learning curve. Of note, surgeon experience with minimally invasive AVR was not correlated with CUSUM results. In fact, the first 3 surgeons, including 1 with extensive RAMT experience, demonstrated evidence of a learning curve. The last 3 surgeons had no prior experience with RAMT, yet they had patients. selection changed.

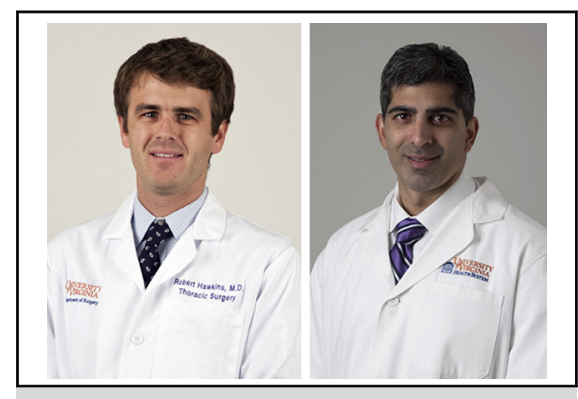

Robert B. Hawkins, MD, and Gorav Ailawadi, MD

Central Message

Whether individual or institutional, experience helps in overcoming the learning curve for minimally invasive approaches. Sutureless valves can make minimally invasive approaches more widespread.

See Article page 1537.

no evidence of a learning curve. The overall institutional experience with sutureless AVR via RAMT appears to have minimized the learning curve for the latter 3 surgeons.

Although this study concluded there is no significant learning curve for RAMT sutureless AVR, the clear confounder is the most experienced surgeon who had gone through his learning curve before training others. It is clear the institutional experience helped ensure that new surgeons performing this approach did not have detrimental outcomes. It would be unrealistic to extrapolate these outcomes to any center beginning RAMT, with or without sutureless valve experience. Nevertheless, sutureless AVR seems to have a role in surgical AVR with the potential for significant advantages. At our institution, many patients have benefited from minimally invasive techniques, and these techniques are an important approach to cardiac surgery. However, because of the complexity, we think specialization within the surgical team is of benefit to

Finally, the CUSUM approach is a powerful tool for detecting variance in expected clinical outcome. Continuous quality improvement is fantastic in theory but difficult to execute. As outlined in this article, ${ }^{1}$ monthly review of CUSUM charts is an easy way to visualize outcomes in a risk-adjusted manner. The process works, and in this study suboptimal outcomes were identified early and patient 


\section{References}

1. Murzi M, Cerillo AG, Gilmanov D, Giovanni C, Farneti P, Glauber M, et al. Exploring the learning curve for minimally invasive sutureless aortic valve replacement. J Thorac Cardiovasc Surg. 2016;152:1537-46.

2. Holzhey DM, Seeburger J, Misfeld M, Borger MA, Mohr FW. Learning minimally invasive mitral valve surgery: a cumulative sum sequential probability analysis of 3895 operations from a single high-volume center. Circulation. 2013;128:483-91.
3. Murzi M, Cerillo AG, Bevilacqua S, Gilmanov D, Farneti P, Glauber M. Traversing the learning curve in minimally invasive heart valve surgery: a cumulative analysis of an individual surgeon's experience with a right minithoracotomy approach for aortic valve replacement. Eur J Cardiothorac Surg. 2012;41:1242-6.

4. Gilmanov D, Miceli A, Ferrarini M, Farneti P, Murzi M, Solinas M, et al. Aortic valve replacement through right anterior minithoracotomy: can sutureless technology improve clinical outcomes? Ann Thorac Surg. 2014;98:1585-92. 